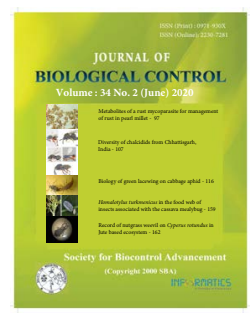

Research Article

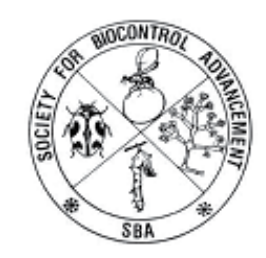

\title{
Parasitic wasps of the family Encyrtidae Walker (Hymenoptera: Chalcidoidea) from Dadra and Nagar Haveli, India
}

\author{
A. RAMESHKUMAR*, S. I. KAZMI and B. MAJUMDER \\ Zoological Survey of India, M-Block, New Alipore, Kolkata - 700053, West Bengal, India \\ *Corresponding author E-mail: drrameshtrichy@gmail.com
}

ABSTRACT: Twenty-one encyrtid species under 15 genera belonging to the family Encyrtidae (Hymenoptera: Chalcidoidea) are reported for the first time from Dadra and Nagar Haveli (Union Territory), India along with their hosts and distribution details.

KEY WORDS: Encyrtidae, parasitoids, biocontrol, new distributional records, Union Territory

(Article chronicle: Received: 27-02-2020; Revised: 15-05-2020; Accepted: 21-05-2020)

\section{INTRODUCTION}

The family Encyrtidae Walker is one of the largest families of the superfamily Chalcidoidea and easiest to recognize by the enlarged mesopleuron (Hayat 2006; Noyes 2004). The members of the family are the primary internal parasitoids of insects as well as arachnids and hyperparasitoids through other hymenopterous parasitoids (Noyes et al. 1997). Most of the encyrtid parasitoids attack hemipterans viz., Coccidae, Pseudococcidae, Diaspididae, Aphidoidea, Psylloidea, Kerridae, Eriococcidae etc. (Hayat 2006). They also parasitize eggs or larvae of beetles (Coleoptera), lacewings (Neuroptera), bugs (Hemiptera), butterflies and moths (Lepidoptera), flies (Diptera), grasshoppers (Orthoptera), ootheca of cockroaches (Blattodea), nymphs of ticks, spider eggs and mites (Arachnida). On the other hand, some species are polyembryonic in nature while some species are hyperparasitoids (secondary parasitoids) via other Encyrtidae, Pteromalidae, Aphelinidae, Braconidae, Dryinidae etc. (Hayat 2006). The family Encyrtidae is represented by 742 species from India whereas 1111 species are reported from the Oriental region (Noyes 2019). The keys to Indian genera and species with associated data like synonymy, host, distribution etc., were provided by Hayat (2006).

Dadra and Nagar Haveli is a Union Territory (occupying about $491 \mathrm{~km}^{2}$ ) in India, lying between $20^{\circ} 0^{\prime}$ to $20^{\circ} 25^{\prime} \mathrm{N}$ and $72^{\circ} 50^{\prime}$ to $73^{\circ} 15^{\prime} \mathrm{E}$. Only less than 500 insect species were reported earlier by Chandra (2011). Kazmi et al. (2003) recorded eight species of butterflies, Ghosh et al. (2018) studied the aquatic beetle fauna and Chandra et al. (2018) studied the diversity of hemipteran fauna. No comprehensive studies on the encyrtid fauna from Dadra \& Nagar Haveli have so far been made to estimate the possible number of taxa inhabiting the area. Although, the present study provides a list of the encyrtid fauna from collections, thorough surveys are required to chalk out strategies to collect more information.

\section{MATERIAL AND METHODS}

Encyrtid parasitoids were collected through net sweeping and yellow pan trap methods from different areas of Dadra and Nagar Haveli. Specimens were processed and mounted as per standard protocol (Noyes 1982) and identified up to species level through appropriate keys. Voucher specimens are deposited in National Zoological Collection, Zoological Survey of India, Kolkata (NZSI), India.

\section{RESULTS AND DISCUSSION}

Twenty-one species under 15 genera of the family Encyrtidae were identified and are reported for the first time from Dadra and Nagar Haveli Union territory, India. Specimens of the genus Metaphycus Mercet (new to Dadra and Nagar Haveli) were also collected, but are not yet determined to species. 


\section{Acerophagus papayae Noyes and Schauff}

Acerophagus papayae Noyes and Schauff 2003, 105

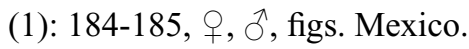

Specimen examined: India, Dadra and Nagar Haveli, Dudhni garden $\left(20.18123^{\circ} \mathrm{N}, 073.15342^{\circ} \mathrm{E}\right), 01$ male, yellow pan trap, 25.i.2018 (A. Rameshkumar).

Host: Paracoccus marginatus (Noyes and Schauff, 2003)

Distribution: India: Andhra Pradesh, Karnataka, Tamil Nadu, Puducherry, Dadra and Nagar Haveli (New record).

\section{Agarwalencyrtus citri (Agarwal)}

Coccidencyrtus citri Agarwal 1965: 75, 76-77, , Holotype: India, Aligarh.

Agarwalencyrtus citri (Agarwal): Hayat, 1981: 15-16, $\uparrow$, tax., des., Aligarh, Kolyatji near Bikaner, Bikaner and Nagaur rec.

Specimen examined: India, Dadra and Nagar Haveli, Dudhni garden $\left(20.18123^{\circ} \mathrm{N}, 073.15342^{\circ} \mathrm{E}\right), 01$ female, yellow pan trap, 25.i.2018 (A. Rameshkumar).

Hosts: Planococcus citri (Agarwal 1965); Tomosvaryella sp. (Diptera) (Hayat 1986).

Distribution: India: Andhra Pradesh, Assam, Bihar, Delhi, Jharkhand, Kerala, Meghalaya, Rajasthan, Tamil Nadu, Uttar Pradesh, Dadra and Nagar Haveli (New record)

\section{Anagyrus aquilonaris (Noyes and Hayat)}

Cremesina aquilonaris Noyes and Hayat 1984: 261-262, +, $\widehat{\jmath}$, India, Aligarh.

Anagyrus aquilonaris (Noyes and Hayat): Shamim and Shafee, 1989: 45.

Specimen examined: India, Dadra and Nagar Haveli, Dudhni garden $\left(20.18123^{\circ} \mathrm{N}, 073.15342^{\circ} \mathrm{E}\right), 01$ female, yellow pan trap, 25.i.2018 (A. Rameshkumar).

Hosts: Unknown.

Distribution: India: Andhra Pradesh, Assam, Bihar, Delhi, Jammu \& Kashmir, Jharkhand, Karnataka, Kerala, Tamil Nadu, Uttarakhand, Uttar Pradesh, Dadra and Nagar Haveli (New record).

\section{Anagyrus dactylopii (Howard)}

Aphycus dactylopii Howard 1898: 242, †, China, Hong Kong.

Anagyrus dactylopii (Howard): Timberlake, 1924: 224.

Specimen examined: India, Dadra and Nagar Haveli, Khanvel $\left(20.13060^{\circ} \mathrm{N}, 073.06226^{\circ} \mathrm{E}\right), 01$ female, yellow pan trap, 20.i.2018 (A. Rameshkumar).

Hosts: Ferrisia virgata on Acacia sp. (Subba Rao and Rai 1970), same host on pomelo (Noyes and Hayat 1994); Maconellicoccus hirsutus on Anona squamosa and grape (Mani and Krishnamurthy 1989); Nipaecoccus viridis (Shafee and Avasthi 1983), same host on Citrus vines, Euphorbia hirta and Dalbergia sisso (Subba Rao et al. 1965); Casuarina sp., Tephrosia purourea (Noyes and Hayat 1994) Planococcus citri on Cirtus medca (Agarwal 1965); Rastrococcus cappariae (Shafee and Avasthi 1983).

Distribution: India: Andaman \& Nicobar Islands, Andhra Pradesh, Assam, Bihar, Delhi, Goa, Himachal Pradesh, Jammu \& Kashmir, Jharkhand, Karnataka, Kerala, Maharashtra, Odisha, Punjab, Tamil Nadu, Uttar Pradesh, Uttarakhand, Dadra and Nagar Haveli (New record).

\section{Anagyrus gracilis (Hayat)}

Doliphoceras gracilis Hayat 1970: 114-115, ․ India, Bannerghatta N. P.

Anagyrus gracilis (Hayat): Noyes and Hayat, 1994: 75, 95-96, + , ô, des., key, recorded from several localities in India: also Madagascar and Nepal.

Specimen examined: India, Dadra and Nagar Haveli, Dudhni garden $\left(20.18123^{\circ} \mathrm{N}, 073.15342^{\circ} \mathrm{E}\right), 01$ female, yellow pan trap, 25.i.2018 (A. Rameshkumar).

Hosts: Brevennia rehi (Nalini and Manickavasagam 2011), Coccidohystrix insolita (Noyes and Hayat 1994), Antigastra catalaunalis (Hayat 2006).

Distribution: India: Andaman and Nicobar Islands, Andhra Pradesh, Assam, Bihar, Delhi, Himachal Pradesh, Jammu \& Kashmir, Jharkhand, Karnataka, Kerala, Maharashtra, Odisha, Rajasthan, Tamil Nadu, Uttar Pradesh, West Bengal, Dadra and Nagar Haveli (New record).

\section{Anagyrus thailandicus (Myartseva)}

Anathrix thailandicus Myartseva 1979: 1746-1747, o, Thailand, Muang 
Anagyrus thalilandicus (Myartseva): Noyes, 2000: 29.

Specimen examined: India, Dadra and Nagar Haveli, Khanvel $\left(20.13060^{\circ} \mathrm{N}, 073.06226^{\circ} \mathrm{E}\right), 01$ female, yellow pan trap, 20.i.2018 (A. Rameshkumar).

Hosts: Unknown.

Distribution: India: Assam, Kerala, Tamil Nadu, West Bengal, Dadra and Nagar Haveli (New record).

\section{Anicetus angustus Hayat, Alam and Agarwal}

Anicetus angustus Hayat, Alam and Agarwal 1975: 31, 35, , India, Goa, Madgao.

Specimens examined: India, Dadra and Nagar Haveli, Dudhni garden $\left(20.18123^{\circ} \mathrm{N}, 073.15342^{\circ} \mathrm{E}\right), 02$ females, yellow pan trap, 25.i.2018 (A. Rameshkumar).

Hosts: Ceroplastes actiniformis (Hayat 1986).

Distribution: India: Goa, Dadra and Nagar Haveli (New record).

\section{Apoleptomastix bicoloricornis (Girault)}

Leptomastix bicoloricornis Girault 1915: 152-153, ㅇ, Australia, Cairns, Gordonvale.

Apoleptomastix bicoloricornis (Girault): Kerrich, 1982: 427

Specimen examined: India, Dadra and Nagar Haveli, Dudhni garden $\left(20.18123^{\circ} \mathrm{N}, 073.15342^{\circ} \mathrm{E}\right), 01$ female, yellow pan trap, 25.i.2018 (A. Rameshkumar).

Hosts: Coccidohystrix insolita (Shamim and Shafee 1984), Brevennia rehi, Heterococcus nigeriensis (Noyes and Hayat 1994).

Distribution: India: Andhra Pradesh, Bihar, Delhi, Jammu \& Kashmir, Jharkhand, Karnataka, Kerala, Maharashtra, Puducherry, Rajasthan, Tamil Nadu, Uttar Pradesh, West Bengal, Dadra and Nagar Haveli (New record).

\section{Callipteroma sexguttata Motschulsky}

Callipteroma sexguttata Motschulsky 1863: 37, ふૈ, Sri Lanka, Mt. Patannas.

Specimens examined: India, Dadra and Nagar Haveli, Madhuban Dam $\left(20.17879^{\circ} \mathrm{N}, 073.06289^{\circ} \mathrm{E}\right), 01$ female,
Sweep Net, 22.1.2018; Khanvel (20.13060 ${ }^{\circ}$, 073.06226 $\left.{ }^{\circ} \mathrm{E}\right)$, 02 females, yellow pan trap, 20.i.2018 (A. Rameshkumar).

Hosts: Heliococcus summervillei (Gupta and Joshi 2013).

Distribution: India: Andaman \& Nicobar Islands, Delhi, Karnataka, Kerala, Madhya Pradesh, Maharashtra, Odisha, Rajasthan, Tamil Nadu, Uttar Pradesh, Dadra and Nagar Haveli (New record).

10. Cheiloneurus bangalorensis (Subba Rao)

Chrysopophagus bangalorensis Subbo Rao 1957: 382383, ㅇ, ô, India, Bangalore.

Cheiloneurus bangalorensis (Subba Rao): Hayat, 1972: 212, ㅇ, ô, tax., Aligarh rec.

Specimen examined: India, Dadra and Nagar Haveli, Dudhni garden $\left(20.18123^{\circ} \mathrm{N}, 073.15342^{\circ} \mathrm{E}\right) 01$ female, yellow pan trap, 25.i.2018 (A. Rameshkumar).

Hosts: Antonina sp. on grass (Hayat 1972); A. graminis (Anis and Hayat 2002).

Distribution: India: Andaman \& Nicobar Islands, Andhra Pradesh, Assam, Karnataka, Kerala, Rajasthan, Tamil Nadu, Uttarakhand, Uttar Pradesh, West Bengal, Dadra and Nagar Haveli (New record).

\section{Cheiloneurus exitiosus (Perkins)}

Echthrogonatopus exitiosus Perkins 1906: 256. .

Cheiloneurus exitiosus (Perkins): Guerrieri and Viggiani, 2005: 314, hosts, distrib., tax., rec.

Specimen examined: India, Dadra and Nagar Haveli, Khanvel $\left(20.13060^{\circ} \mathrm{N}, 073.06226^{\circ} \mathrm{E}\right), 01$ female, yellow pan trap, 20.i.2018 (A. Rameshkumar).

Hosts: Cnaphalocrocis medinalis and Marasmia exigua (Behera et al. 2002).

Distribution: India: Andhra Pradesh, Assam, Bihar, Karnataka, Kerala, Maharashtra, Meghalaya, Mizoram, Odisha, Puducherry, Tamil Nadu, Uttarakhand, Uttar Pradesh, West Bengal, Dadra and Nagar Haveli (New record).

12. Cheiloneurus latifrons Hayat, Alam and Agarwal

Cheiloneurus latifrons Hayat, Alam and Agarwal 1975: 46-48, +, India, Karnataka, Hebbal. 
Specimen examined: India, Dadra and Nagar Haveli, Dudhni garden $\left(20.18123^{\circ} \mathrm{N}, 073.15342^{\circ} \mathrm{E}\right), 01$ female, yellow pan trap, 25.i.2018 (A. Rameshkumar).

Hosts: Phenacoccus indicus (Noyes and Hayat 1994), Peliococcus indicus (Hayat 1986).

Distribution: India: Delhi, Himachal Pradesh, Karnataka, Kerala, Tamil Nadu, Uttarakhand, Uttar Pradesh, Dadra and Nagar Haveli (New record).

\section{Copidosoma floridanum (Ashmead)}

Berecyntus floridanus Ashmead 1900: 365, ㅇ, USA, Florida, Biscayne Bay.

Specimens examined: India, Dadra and Nagar Haveli, Dudhni garden $\left(20.18123^{\circ} \mathrm{N}, 073.15342^{\circ} \mathrm{E}\right), 04$ females, yellow pan trap, 25.i.2018 (A. Rameshkumar).

Hosts: Plusia signata (Hayat 1986), Argyrogramma signatum, Helicoverpa armigera (Kazmi and Hayat 2012), Cosmophila sabulifera (Hayat 2006), Chrysodeixis eriosoma, C. chalcites (Noyes and Hayat 1994).

Distribution: India, Bihar, Jammu \& Kashmir, Jharkhand, Karnataka, Kerala, Odisha, Tamil Nadu, Uttar Pradesh, West Bengal, Dadra and Nagar Haveli (New record).

\section{Encyrtus infelix (Embleton)}

Comys infelix Embleton 1902: 223 (foot note). Lectotype 우 [designated by Graham, 1969:308]

Encyrtus infelix (Embleton): Peck, 1963: 356-357.

Specimens examined: India, Dadra and Nagar Haveli, Vasona $\left(20.20292^{\circ} \mathrm{N}, 073.02486^{\circ} \mathrm{E}\right) 02$ females and 04 males, yellow pan trap, 23.i.2018 (A. Rameshkumar).

Hosts: Saissetia sp. (Gupta and Poorani 2009), Coccus hesperidum (Konar and Saha 2002).

Distribution: India, Assam, Karnataka, Tamil Nadu, West Bengal, Dadra and Nagar Haveli (New record).

\section{Homalotylus albiclavatus (Agarwal)}

Neoaenasioidea albiclavatus Agarwal 1970: 27, + , India, Aligarh.

Homalotylus albiclavatus (Agarwal): Hayat, Alam and Agarwal, 1975: 69, ㅇ, ठ̂, key, hosts, distrib., Shencottah,
Shoranur, Joginder Nagar and Lakhimpur Kheri rec.

Specimen examined: India, Dadra and Nagar Haveli, Khanvel $\left(20.13060^{\circ} \mathrm{N}, 073.06226^{\circ} \mathrm{E}\right), 01$ female, yellow pan trap, 20.i.2018 (A. Rameshkumar).

Hosts: Nipaecoccus viridis, Pseudococcus sp., Pullus sp. (Kazmi and Hayat 2009).

Distribution: India, Himachal Pradesh, Jharkhand, Karnataka, Kerala, Rajasthan, Tamil Nadu, Uttar Pradesh, West Bengal, Dadra and Nagar Haveli (New record).

\section{Leptomastix nigrocincta Risbec}

Leptomastix nigrocincta Risbec 1959 (1958): 27, ㅇ, Madagascar.

Specimen examined: India, Dadra and Nagar Haveli, Khanvel $\left(20.13060^{\circ} \mathrm{N}, 073.06226^{\circ} \mathrm{E}\right), 01$ female, yellow pan trap, 20.i.2018 (A. Rameshkumar).

Hosts: Coccidohystrix insolita (Sankaran 1959), Phenacoccus sp. (Gupta and Joshi 2013).

Distribution: India: Andaman and Nicobar Islands, Andhra Pradesh, Delhi, Karnataka, Kerala, Madhya Pradesh, Puducherry, Tamil Nadu, Uttar Pradesh, Dadra and Nagar Haveli (New record).

\section{Microterys nietneri (Motschulsky)}

Encyrtus nietneri Motschulsky 1863: 55, ㅇ. Sri Lanka, Mt. Patanna.

Microterys nietneri (Motschulsky): Trjapitzin and Sugonjaev, 1972: 617.

Specimens examined: India, Dadra and Nagar Haveli, Khanvel $\left(20.13060^{\circ} \mathrm{N}, 073.06226^{\circ} \mathrm{E}\right), 05$ females, yellow pan trap, 20.i.2018 (A. Rameshkumar).

Hosts: Coccus hesperidum (Pruthi and Mani 1945), Parasaissetia nigra (Ayyar and Margabandhu 1934), Saissetia coffeae, Crossandra infundibuliformis (Sankaran and Pattar 1977).

Distribution: India: Karnataka, Kerala, Tamil Nadu, Uttar Pradesh, Dadra and Nagar Haveli (New record).

\section{Neodusmetia sangwani (Subba Rao)}

Dusmetia sangwani Subba Rao 1957: 385-386, ㅇ, ત̂, India, Bangalore. 
Neodusmetia sangwani (Subba Rao): Kerrich, 1964: 76.

Specimen examined: India, Dadra and Nagar Haveli, Vasona $\left(20.20292^{\circ} \mathrm{N}, 073.02486^{\circ} \mathrm{E}\right), 01$ male, yellow pan trap, 23.i.2018 (A. Rameshkumar).

Hosts: Antonina sp. on grass (Hayat 1981), same sp. on Cynodon sp. (Shafee et al. 1975), same sp. on Eragrastes ciliaris (Hayat 1977); A. indica (=graminis) (Subba Rao 1957; Burks 1957).

Distribution: Probably throughout India. India: Andaman \& Nicobar Islands, Andhra Pradesh, Assam, Delhi, Karnataka, Kerala, Puducherry, Rajasthan, Tamil Nadu, Uttar Pradesh, Dadra and Nagar Haveli (New record).

\section{Ooencyrtus segestes Trjapitzin}

Ooencyrtus segestes Trjapitzin 1965: 320-321, q, Indonesia, Komodo Island.

Specimen examined: India, Dadra and Nagar Haveli, Khanvel $\left(20.13060^{\circ} \mathrm{N}, 073.06226^{\circ} \mathrm{E}\right), 01$ female, yellow pan trap, 20.i.2018 (A. Rameshkumar).

Hosts: Unknown.

Distribution: India: Andhra Pradesh, Assam, Bihar, Delhi, Himachal Pradesh, Karnataka, Odisha, Puducherry, Punjab, Tamil Nadu, Uttar Pradesh, Uttarakhand, West Bengal, Dadra and Nagar Haveli (New record).

\section{Tassonia amaura Hayat}

Tassonia amaura Hayat 2003: 233-234, †, India, Mysore, Brindavan Garden

Specimen examined: India, Dadra and Nagar Haveli, Khanvel $\left(20.13060^{\circ} \mathrm{N}, 073.06226^{\circ} \mathrm{E}\right), 01$ female, yellow pan trap, 20.i.2018 (A. Rameshkumar).

Hosts: Unknown.

Distribution: India: Arunachal Pradesh, Karnataka, Odisha, Uttarakhand, Dadra and Nagar Haveli (New record).

\section{Tassonia gloriae Girault}

Tassonia gloriae Girault 1921: 2,, , Australia, Wynnum.

Specimens examined: India, Dadra and Nagar Haveli, Dudhni garden $\left(20.18123^{\circ} \mathrm{N}, 073.15342^{\circ} \mathrm{E}\right), 01$ female, sweep net, 25.i.2018; Khanvel $\left(20.13060^{\circ} \mathrm{N} 073.06226^{\circ} \mathrm{E}\right)$,
02 females, yellow pan trap, 25.i.2018; Khanvel, 01 female, yellow pan trap, 20.i.2018 (A. Rameshkumar).

Hosts: Hysteroneura setariae on Cynodon dactylon (Hayat 1979; Kazmi and Hayat 2009), Longiunguis sacchari on Saccharum officinarum (Kazmi and Hayat 2009; Shafee et al. 1975), same host on Sorghum vulgare (Kazmi and Hayat 2009; Hayat 1979); Myzus persicae (Hayat 2006 and Hayat 1979).

Distribution: India: Andhra Pradesh, Arunachal Pradesh, Bihar, Goa, Daman \& Diu, Himachal Pradesh, Jharkhand, Karnataka, Kerala, Odisha, Punjab, Rajasthan, Tamil Nadu, Uttarakhand, Uttar Pradesh, West Bengal, Dadra and Nagar Haveli (New record).

Encyrtids are extremely important parasitoids because most of the species are involved in biological control of many agricultural as well as horticultural pests. Even though the family is studied well throughout India, Dadra and Nagar Haveli are not explored. In the present paper, 21 species under 15 genera of encyrtids were recorded for the first time from Dadra and Nagar Haveli. However, further more surveys are required in the agricultural and horticultural belts in these regions which may likely to yield a large number of known and undescribed species.

\section{ACKNOWLEDGMENTS}

The authors are thankful to Dr. Kailash Chandra, Director, Zoological Survey of India, Kolkata for providing necessary facilities to carry out this work. The first author is grateful to Dr. K. Rajmohana, Scientist - D, Mr. Balmohan Baraik, Assistant Zoologist and Mr. Sandeep Kushwaha, Assistant Zoologist for their support during the survey at Dadra and Nagar Haveli.

\section{REFERENCES}

Agarwal MM. 1965. Taxonomy of encyrtid parasites (Hymenoptera: Chalcidoidea) of Indian Coccoidea. Act Hymenopterologica, Fukuoka 2: 37-97.

Agarwal MM. 1970. Some new chalcidoid parasites recorded from Aligarh (India) (Hym., Encyrtidae). Mushi 44(4): $25-29$.

Anis SB, Hayat M. 2002. A revision of the Indian species of Cheiloneurus Westwood (Hymenoptera: Chalcidoidea: Encyrtidae). Orient Insects 36: 129-209. https://doi.org/ $10.1080 / 00305316.2002 .10417330$

Ashmead WH. 1900. On the genera of chalcid-flies belonging to the subfamily Encyrtinae. Proc US Nat Mus. 22: 323412. https://doi.org/10.5479/si.00963801.22-1202.323 
Ayyar TVR, Margabandhu, V. 1934. Further record of Indo-Ceylonese chalcid flies. J Bom Nat Hist Soc. 37: 193-196.

Burks BD. 1957. A new parasite of the Rhodes-Grass scale (Hymenoptera: Encyrtidae). Bull Brooklyn Entomol Soc. 52: 124-127.

Chandra K. 2011. Insect fauna of states and Union Territories in India. In. Arthropods and their Conservation in India (Insects \& Spiders). ENVIS Bull. 14 (1): 189-218.

Chandra K, Bhandari R, Khuswaha S. 2018. Diversity of Hemiptera fauna of Dadra Nagar Haveli Wildlife Sanctuary area, Dadra Nagar Haveli, India. $J$ Nat Res Dev. 13(1): 134-137.

Embleton AL. 1902. On the economic importance of the parasites of Coccidae. Trans Entomol Soc. (London) 35: 219-229.

Ghosh SK, Chakraborty U, Mitra B. 2018. Aquatic beetle fauna of Dadra and Nagar Haveli, Daman \& Diu, India with taxonomic notes on Eretes griseus (Fabricious 1781) of national collection of Zoological Survey of India, J Phamr Innov. 7(11): 439-444.

Girault AA. 1915. Australian Hymenoptera Chalcidoidea VII. The family Encyrtidae with descriptions of new genera and species. Memoirs Queensland Mus. 4: 1-184.

Girault AA. 1921. New Animals of Australia and Old Men of the Earch. 3pp. Brisbane (privately published).

Guerrieri E, Viggiani G. 2005. A review of the encyrtid (Hymenoptera: Chalcidoidea) parasitoids of Dryinidae (Hymenoptera: Chrysidoidea) with description of a new species of Cheiloneurus. Syst Biodivers. 2(3): 305-317. https://doi.org/10.1017/S1477200004001537

Gupta A, Poorani J. 2009. Taxonomic studies on a collection of Chalcidoidea (Hymenoptera) from India with new distribution records. J Threat Taxa 1(5): 301. https://doi. org/10.11609/JoTT.o1861.300-4

Gupta A, Joshi S. 2013. Additions to the fauna of parasitic wasps (Hymenoptera: Chalcidoidea) and Coccoids (Hemiptera: Coccoidea) from the Andaman and Nicobar Islands, India, with illustrations and diagnoses. $J$ Threat Taxa 5(11): 4544. https://doi.org/10.11609/JoTT. o3360.4542-55

Hayat M. 1970. Three new species of Encyrtidae (Hymenoptera, Chalcidoidea) from India. Bull Entomol. 10: $110-115$.
Hayat M. 1972. Descriptions of two new genera and species of Encyrtidae (Hymenoptera, Chalcidoidea), with notes on some described species. Acta Entomol Bohemoslov. 69(3): 207-214.

Hayat M. 1977. A preliminary report on the chalcids (Insecta: Hymenoptera) from Rajasthan, India. Newslett ZSI 3: 45-48.

Hayat M. 1979. Taxonomic notes on Indian Encyrtidae (Hymenoptera: Chalcidoidea) I. J Nat Hist. 13: 315326. https://doi.org/10.1080/00222937900770251

Hayat M. 1981. Taxonomic notes on Indian Encyrtidae (Hym: Chalcidoidea) II. J Nat Hist. 15: 17-29. https:// doi.org/10.1080/00222938100770021a

Hayat M. 1986. A new genus and species of Encyrtidae (Hymenoptera: Chalcidoidea) from India. Colemania $\mathbf{3}$ : 21-23.

Hayat M. 2003. Records and descriptions of Indian Encyrtidae (Hymenoptera: Chalcidoidea). Orient Insects 37: 233234. https://doi.org/10.1080/00305316.2003.10417345

Hayat M. 2006. Indian Encyrtidae (Hymenoptera: Chalcidoidea) pp.198 M. Hayat, Department of Zoology, Aligarh Muslim University, India.

Hayat M, Alam SM, Agarwal MM. 1975. Taxonomic survey of encyrtid parasites (Hymenoptera: Encyrtidae) in India. Aligarh Muslim University Publications (Zoological Series) on Indian Insect Types, 9: i-iii. 1-112.

Howard LO. 1898. On some new parasitic insects of the subfamily Encyrtinae. Proc US Natl Mus. 21: 231-248.

Kazmi SI, Hayat M. 2009. Description of a new species and new record including some known species of Encyrtidae (Hymenoptera: Chalcidoidea) from India. Rec Zool Sur India 109(3): 109-114.

Kazmi SI, Hayat M. 2012. Records of some species of Copidosoma Ratzeburg (Hymenoptera: Encyrtidae) from India, with description of a new species. Rec Zool Sur India 112(1): 96.

Kazmi SI, Arora GS, Bhattacharyya AK, Faisal M. 2003. On a collection of Lepidoptera (Butterfly) fauna in the Desert Regional Station, Zoological Survey of India, Jodhpur. Rec Zool Sur India 101(Part 1-2): 1-18.

Kerrich GJ. 1964. On the European species of Dusmetia Mercet and a new Oriental genus (Hymenoptera: 
Chalcidoidea: Encyrtidae). Entomophaga 9: 75-79. https://doi.org/10.1007/BF02375742

Kerrich GJ. 1982. Further systematic studies on tetracnemine Encyrtidae (Hymenoptera: Chalcidoidea) including a revision of the genus Apoanagyrus Compere. J Nat Hist. 16: 399-430. https://doi. org/10.1080/00222938200770331

Konar A, Saha PK. 2002. Incidence of insect parasite of Ceroplastes floridensis Comstock and C. rubens Maskell attacking orange in Darjeeling of West Bengal. J Interacademicia 6(1): 65-68.

Mani M, Krishnamoorthy A. 1989. Occurrence of mealybugs and their natural enemies on custard apple around Bangalore. J Biol Control 3: 77-81.

Motschulsky Vde. 1863. Essai dúm catalogue des insects de I'lle Ceylon (Suite). Byulleten'Moskovskogo Obshchestva Ispytateley Prirody 36(3): 1-153.

Myartseva SN. 1979. A new spcies of the genus Anathrix (Hymenoptera, Encyrtidae) from Thailand. Zoologicheskii Zhurnal. 58: 1746-1748.

Nalini T, Manickavasagam S. 2011. Records of Encyrtidae (Hymenoptera: Chalcidoidea) parasitoids on mealybugs (Hemiptera: Pseudococcidae) from Tamil Nadu, India. Check List 7(4): 513. https://doi.org/10.15560/7.4.510

Noyes JS. 1982. Collecting and preserving chalcid wasp (Hymenoptera: Chalcidoidea). J Nat Hist. 16: 315-334. https://doi.org/10.1080/00222938200770261

Noyes JS. 2000. Encyrtidae of Costa Rica (Hymenoptera: Chalcidoidea), 1. The subfamily Tetracneminae, parasitoids of mealybugs (Homoptera: Pseudococcidae). Mem Am Entomol Inst. 62: 355pp.

Noyes JS. 2004. Encyrtidae of Coast Rica (Hymenoptera: Chalcidoidea), 2. Metaphycus and related genera, parasitoids of scale insects (Coccidea) and whiteflies (Aleyrodidae). Mem Am Entomol Inst. 73: 459pp.

Noyes JA. 2019. Universal Chalcidoidea Database. World Wide Web electronic publication. http://www.nhm. ac.uk/chalcidoids (accessed on $30^{\text {th }}$ January, 2020)

Noyes JS, Hayat M. 1984. A review of the genera of Indo -Pacific Encyrtidae (Hymenoptera: Chalcidoidea). Bull British Mus (Nat Hist) (Entomol.) 48: 131-395.
Noyes JS, Hayat M. 1994. Oriental mealybug parasitoids of the Anagyrini (Hymenoptera: Encyrtidae) pp. 395 CAB International, Oxon, UK.

Noyes JS, Schauff MR. 2003. New Encyrtidae (Hymenoptera) from papaya mealybug (Paracoccus marginatus Williams and Granara de Willink) (Hemiptera: Sternorhyncha: Pseudococcidae). Proc Entomol Soc Washington 105(1): 184-185.

Noyes JS, Woolley JB, Zolnerowich G. 1997. 'Chapter 8. Encyrtidae pp.170-320. In: Gibson GAP, Huber JT, Woolley JB (Eds.). Annotated keys to the genera of nearctic Chalcidoidea (Hymenoptera). NRC Research press, Ottawa. Ontario, Canada.794pp.

Peck O. 1963. A catalogue of the Nearctic Chalcidoidea (Insecta: Hymenoptera). Can Entomol. 30: 1-1092.

Perkins RCL. 1906. Leaf-hoppers and their natural enemies (VIII). Bull Hawaiian Sugar Planters'Assoc Exper Sta (Entomol Ser.) 1: 239-267.

Pruthi HS, Mani MS. 1945. Our knowledge of the insect and mite pests of citrus in India and their control. Imperial Council of Agricultural Research, Scientific Monograph, No.16: 42 pp.

Risbec J. 1958. Encyrtidae de Madagascar (Hym. Chalcid.). Bull de I'Acad Malgache 35: 17-44.

Shamim SM, Shafee SA. 1984. Four new species of Encyrtidae (Hymenoptera) from Bihar, India. Indian $J$ Syst Entomol. 1: 23-28.

Sankaran T. 1959. A note on the two parasites of Phenacoccus insolitus Green (Hemiptera: Coccidae). Proc Royal Entomol Soc Lon (A) 34: 25-26. https://doi. org/10.1111/j.1365-3032.1959.tb00225.x

Sankaran T, Patter GL. 1977. Some parasites of Parasaissetia nigra and Saissetia spp. (Homoptera: Coccidae) from south India. Commonwealth Institute of Biological Control. Tech Bull. 18: 145-150.

Shafee SA, Avasthi RK. 1983. First record of Clausenia Ishii (Hymenoptera: Chalcidoidea) from India with descriptions of two new species. J Bom Nat Hist Soc. 80: 176-179.

Shafee SA, Alam SM, Agarwal MM. 1975. Taxonomic survey of encyrtid parasites (Hymenoptera: Encyrtinae) in India, Aligarh Muslim University Publications (Zoological Series) on Indian insect Types 10: iii+1$125 \mathrm{pp}$. 
RAMESHKUMAR et al.

Shamim SM, Shafee SA. 1989. Four species of Tetracneminae (Hymenoptera: Encyrtidae) from north India. Indian $J$ Syst Entomol. 6: 41-46.

Subba Rao BR. 1957. Some new species of Hymenoptera. Proc Indian Acad Sci. (B) 46: 376-390.

Subba Rao BR, Rai PS. 1970. Studies on the Indian species of the genus Anagyrus Howard, 1896 (Hymenoptera: Encyrtidae). Beitrage zur Entomologie 20: 85-96.

Subba Rao BR, Sangwan HS, Abbasi QA, Singh Y, Ksheersagar AM. 1965. New records of hymenopterous parasites of Nipaecoccus vastor Maskell (Hemiptera:
Coccidae) a serious pest of Citrus spp. from India. Indian J Entomol. 27: 109-110.

Timberlake PH. 1924. The parasites of Pseudococcus maritimus (Ehrb.) in California (Hymenoptera: Chalcidoidea). Part 1. Taxonomic studies. Univ California Pub Entomol. 3: 223-251.

Trjapitzin VA. 1965. Contributions to the knowledge of the encyrtid fauna of the Comodo and Padar Islands with a catalogue of Indonesian species (Hymenoptera, Encyrtidae). Treubia 26: 309-327. 